\title{
Psoriasin (S100A7) is a principal antimicrobial peptide of the human tongue
}

\author{
JE Meyer ${ }^{1,5}$, J Harder $^{2,5}$, B Sipos ${ }^{3}$, S Maune ${ }^{4}$, G Klöppel $^{3}$, J Bartels ${ }^{2}$, J-M Schröder ${ }^{2}$ and R Gläser ${ }^{2}$
}

The human tongue is particularly resistant to bacterial infections although the mouth is continuously exposed to a complex and abundant ensemble of microbes, such as the common intestinal bacterium Escherichia coli. We show that lingual epithelia produce and release, as a primary E. coli-killing compound, the $\mathrm{S} 100$ protein psoriasin. No significant reduction in psoriasin release could be achieved through repeated rinsing of the epithelial surface of the tongue. Psoriasin is produced in the upper layers of the lingual epithelia but is lacking in the most superficial and basal cells. It displays a gradient pattern of expression with decreasing expression from the anterior one-third to the posterior portion of the tongue. Thus, psoriasin may be the key to the resistance of the human tongue toward $E$. coli.

\section{INTRODUCTION}

The human tongue contains a dense mucosa that is constantly colonized by a complex and abundant ensemble of microbes, including bacteria, fungi, and viruses. ${ }^{1-3}$ Despite frequent injuries of the tongue, this surprisingly rarely leads to invasive infections. The mucus acts as a physical barrier that separates the mucosal lining from the environment, and the continual swallowing of saliva clears the mucosal surfaces. This physical barrier is difficult for microorganisms to penetrate and explains, at least in part, the rare infection rates.

In addition, by secretion of chemical factors, the mucosal compartment also has an additional role in innate host defense. Mucosal cells actively respond in an interactive manner: (i) they secrete chemokines and other cytokines to alert and attract various cell types ${ }^{4}$ and (ii) they produce natural antimicrobial peptides in response to microbial products or proinflammatory cytokines. ${ }^{5,6}$ First in 1995 , Schonwetter et al. ${ }^{7}$ reported the isolation of an antimicrobial peptide termed lingual antimicrobial peptide from cattle. Lingual antimicrobial peptide belongs to the group $\beta$-defensins, small cationic peptides that exhibit a broad spectrum of antimicrobial activity. ${ }^{8}$ Lingual antimicrobial peptide is expressed in the bovine tongue epithelia and shows a marked induction of mRNA expression in epithelia surrounding areas of inflammation. The expression of lingual antimicrobial peptide is induced by bacterial lipopolysaccharide and proinflammatory cytokines. ${ }^{9}$
Although little attention has been paid to the possible role of human defensins in the innate host defense of the human tongue, it is likely that human oral tissues produce antimicrobial peptides similar to those found in other macroorganisms. Recent reports provided first limited evidence of human $\beta$-defensin (hBD)-1 and hBD-2 in maintaining oral health, ${ }^{10}$ and Candida-static calprotectin (a heterodimer consisting of the S100 proteins S100A8 and A9) demonstrated a diffuse expression pattern in the human tongue. ${ }^{11}$

Here we describe the identification of the $11,366 \mathrm{Da}$ S100 protein psoriasin (S100A7c) as the dominating Escherichia coli-killing antimicrobial protein of the healthy human tongue, present and secreted on oral mucosal surface and differentially produced in healthy lingual epithelial cells.

\section{RESULTS}

Psoriasin is a principal antimicrobial peptide of the human tongue

We analyzed extracts of epithelial tissue layers of human tongues derived from cadavers for antimicrobial activity and consistently found high titers of E. coli-killing activity. The majority of antimicrobial activity bound to a heparin affinity column. Separation of tongue extracts by preparative reversed-phase (C8) highperformance liquid chromatography (HPLC) and analyses of the resulting fractions for bactericidal activity identified prominent E. coli-killing activity eluting at $60 \%$ acetonitrile (Figure 1).

\footnotetext{
${ }^{1}$ Department of Otorhinolaryngology, Head and Neck Surgery, University of Luebeck, Luebeck, Germany. ${ }^{2}$ Clinical Research Unit, Department of Dermatology, Christian-Albrechts-University, Kiel, Germany. ${ }^{3}$ Department of Pathology, Christian-Albrechts-University, Kiel, Germany. ${ }^{4}$ Department of Otorhinolaryngology, Head and Neck Surgery, Krankenhaus-Holweide, Cologne, Germany. ${ }^{5}$ These authors contributed equally to this manuscript. Correspondence: JE Meyer (jens.meyer@hno.uni-luebeck.de) 
Mass spectrometric analyses by electrospray ionization-mass spectrometry of the fraction containing dominating E. colikilling activity revealed a molecular mass of $11,366 \mathrm{Da}$ (data not shown). This mass is identical to the mass of natural skinderived N-terminally acetylated psoriasin (S100A7). ${ }^{12}$ Peptide mass fingerprinting analysis confirmed the identity of psoriasin.

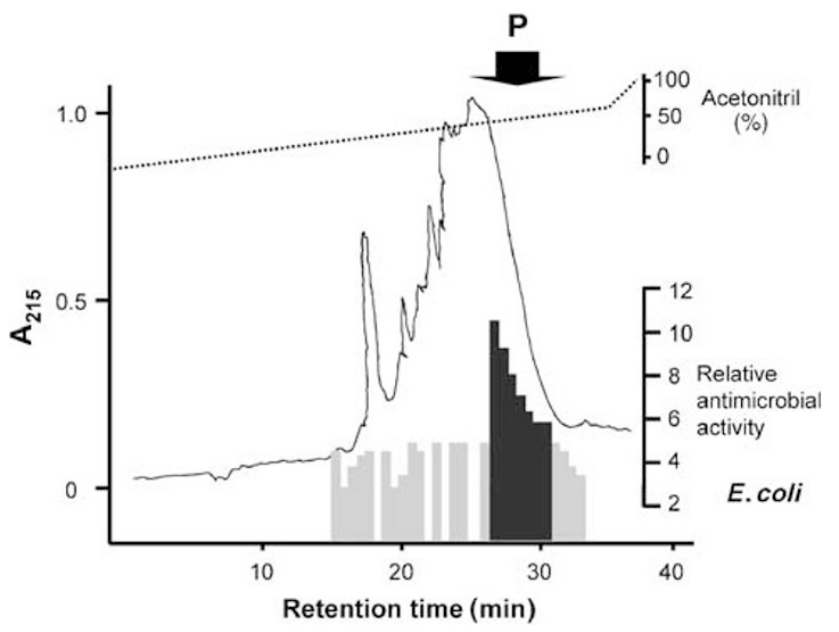

Figure 1 Purification and isolation of psoriasin. Protein extracts of epithelial tissue layers of human tongue derived from cadavers were purified by reversed-phase (RP)-8 HPLC and the resulting fractions were analyzed for Escherichia coli-killing activity (bars). Fractions containing the highest titer E. coli-killing activity (P, black bars) were further subjected to ESI-MS analyses. Multiple charged signals correspond to a mean neutral mass of $11,366 \mathrm{Da}$ (calculated neutral mass of $\mathrm{N}$-acetylated psoriasin $=11,365.8 \mathrm{Da}$ ). ESI-MS, electrospray ionizationmass spectrometry; HPLC, high-performance liquid chromatography.
We were able to purify approximately $40-50 \mu \mathrm{g}$ psoriasin per g tongue tissue.

\section{Psoriasin displays a gradient pattern of expression in the healthy human tongue}

To investigate the distribution of psoriasin in healthy lingual mucosa, we performed immunohistochemistry using a psoriasin monoclonal antibody. Psoriasin staining was reduced from the anterior part to the posterior part of the tongue (Figure 2a-d). Intense intracytoplasmic, but also membrane-bound psoriasin staining (Figure 3a and $\mathbf{b}$ ) was identified in mucosal cells of healthy tongues mainly in the upper, more differentiated layers with weaker staining of mucosal cells in the suprabasal layers (Figure 3a and b). Interestingly, most basal and superficial mucosal cells were lacking psoriasin immunoreactivity whereas intensive psoriasin immunoreactivity was also seen in cells of the lingual crypts (Figure 2b). These results indicate that psoriasin is produced in the tongue epithelium.

\section{Psoriasin is released in vivo}

To address the hypothesis that psoriasin is possibly released in vivo, we analyzed tongue-washing fluids of healthy individuals for the presence of psoriasin (Figure 4a). Individual psoriasin concentrations ranged from 0.6 to $464 \mathrm{ng}$ per $\mathrm{cm}^{2}$ tongue surface $\left(n=20\right.$, median concentration $=1.86 \mathrm{ng}$ per $\mathrm{cm}^{2}$ tongue surface). Comparable amounts were recovered at all investigated time-points (median concentration $=1.75-2.01 \mathrm{ng}$ per $\mathrm{cm}^{2}$ tongue surface). Interestingly, psoriasin concentration was not markedly reduced after repeatedly rinsing the same area (Figure $\mathbf{4 b}$ ). The presence of psoriasin in the tongue-washing
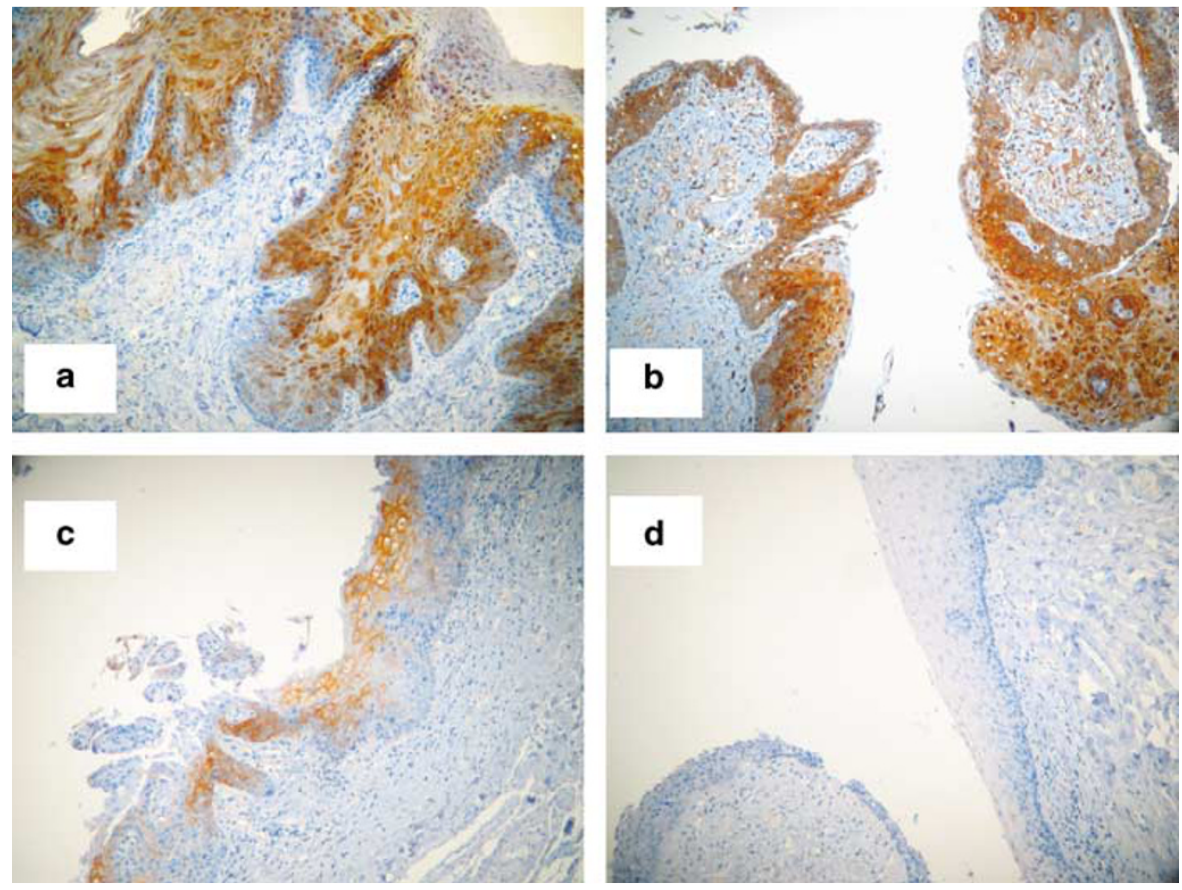

Figure 2 Psoriasin displays a gradient pattern of expression from the tip to the base of the tongue. Psoriasin expression pattern was visualized by immunohistochemistry using a monoclonal antibody directed against psoriasin. (a) tip; (b) free edge; (c) center; (d) base of the tongue. 

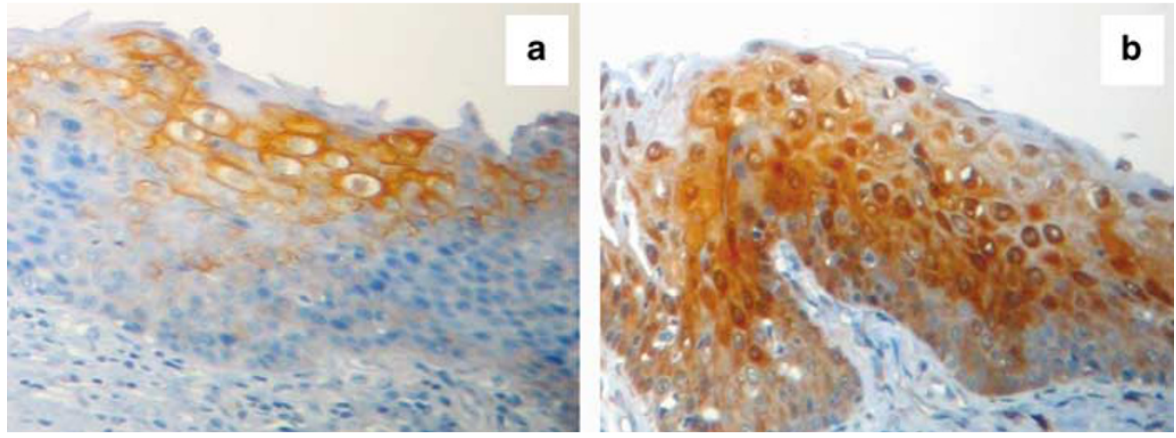

Figure 3 Psoriasin is abundant in the upper layers of the human tongue epithelium, lacking positive staining of the most superficial cells. Psoriasin expression pattern was visualized by immunohistochemistry using a monoclonal antibody directed against psoriasin. Intense intracytoplasmic, but also membrane-bound psoriasin staining was identified mainly in the upper, more differentiated layers of the healthy tongue with the absence of psoriasin in the most superficial cells. (a) Section magnification of Figure 2c; (b) section magnification of Figure $\mathbf{2 b}$.
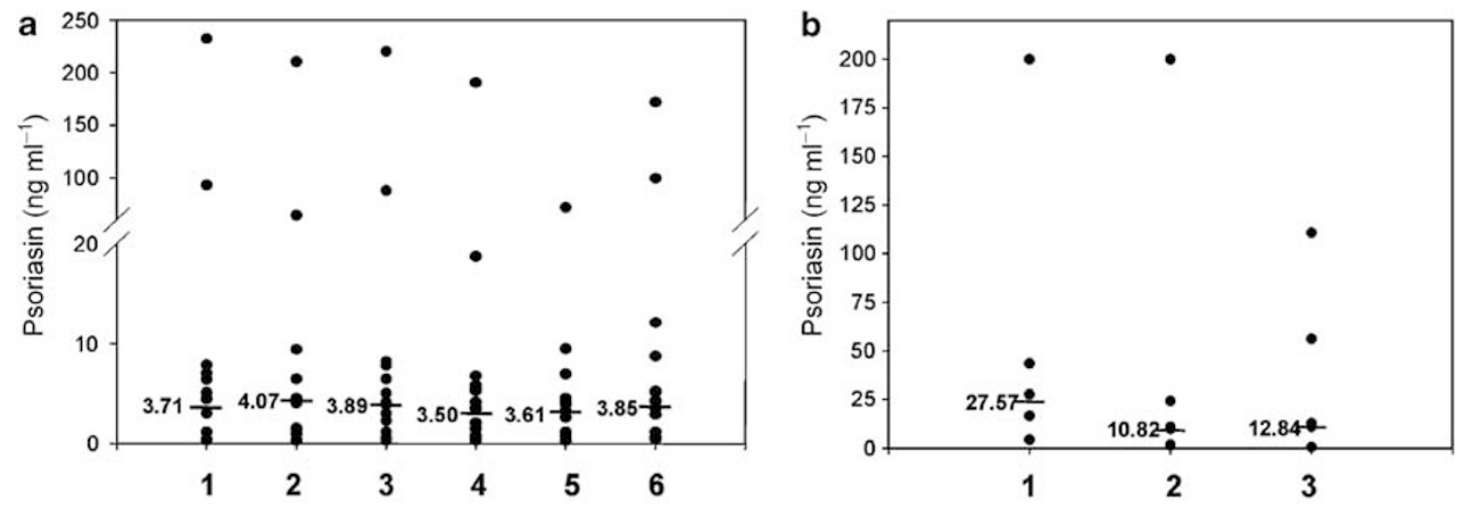

c
Rinsing fluids
Psoriasin $\left(\mathrm{ng} \mathrm{ml}^{-1}\right)$

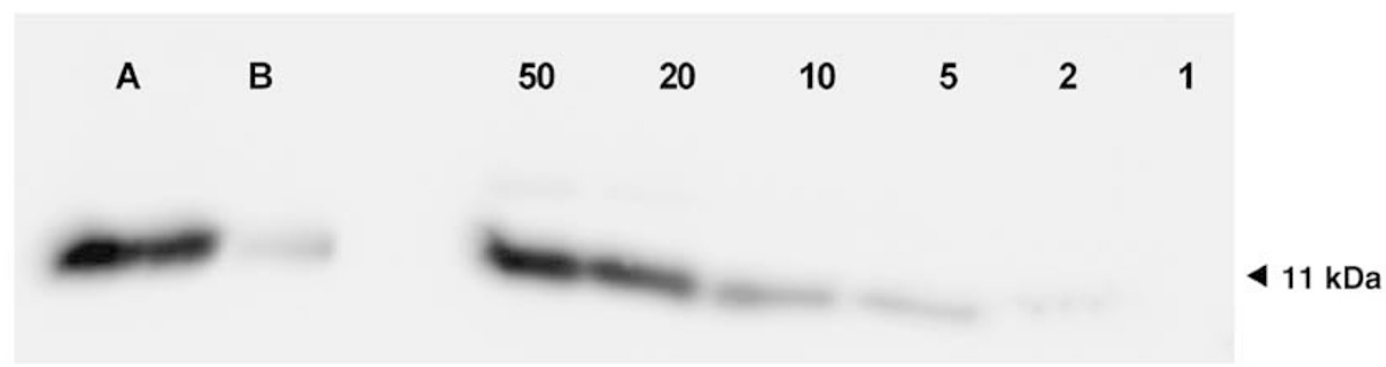

Figure 4 In vivo secretion of psoriasin on the lingual surface. A standardized area $\left(50 \mathrm{~mm}^{2}\right)$ of the tongue was rinsed repeatedly with $900 \mu \mathrm{l} 10 \mathrm{mM}$ sodium phosphate buffer $(\mathrm{pH} 7.4)$ and psoriasin concentration was measured by enzyme-linked immunosorbent assay. (a) The tongues of 20 volunteers were rinsed $30 \mathrm{~min} \mathrm{(1),} 1 \mathrm{~h} \mathrm{(2),} 2 \mathrm{~h} \mathrm{(3),6h} \mathrm{(4),} 12 \mathrm{~h}(5)$, and $24 \mathrm{~h}(6)$ after irrigating the mouth three times. (b) Five volunteers were rinsed 20 times repeatedly in the same area and psoriasin concentration was determined after the first (1), tenth (2), and twentieth (3) rinsing procedure. (c) One milliliter of rinsing fluid was derived from two individuals, subject 1 (A) and 2 (B). Specific detection of psoriasin in the tongue rinsing fluids was verified by western blot analysis using a monoclonal antibody directed against psoriasin. Natural skin-derived psoriasin served as control.

fluids was verified by western blot analyses with representative tongue-washing fluids (Figure 4c).

\section{DISCUSSION}

The human mouth is an area of high microbial density ${ }^{3}$ and frequently occurring injuries. Despite these constant microbial threats, the human tongue is highly resistant against microbial colonization and infection by oral intake. E. coli acts as an indicator organism for the microbiological quality of food and beverages. ${ }^{13}$ Moreover, E. coli is not a normal member of the oral flora, and lingual infections with E. coli are rarely seen, despite daily exposure of $E$. coli strains from animal reservoirs, environmental vehicles, food processing environments, and nutriments through the mouth. ${ }^{14-16}$ The reason for the natural resistance of tongue against $E$. coli infection is not understood. Therefore, we systematically analyzed human tongue for the presence of E. coli-killing factors and identified psoriasin (S100A7) as a dominating antimicrobial component of the healthy human tongue.

Psoriasin was originally discovered in psoriatic skin lesions as a new $\mathrm{Ca}^{2+}$-binding S100 protein. ${ }^{17}$ The S100 proteins (so called 
for their solubility in ammonium sulphate) comprise a multigene family of low molecular weight proteins that are believed to mediate a variety of functions in eukaryotic cells including differentiation, cell-cycle progression, intracellular $\mathrm{Ca}^{2+}$ signaling, and cytoskeletal membrane interactions as well as playing a role in leukocyte chemotaxis. ${ }^{18,19}$ Recently, it was shown that S100 proteins may play a putative role in the innate host defense. Calprotectin exhibits $\mathrm{Zn}^{2+}$-sensitive Candida albicans-selective biostatic activity ${ }^{20,21}$ and follows a diffuse expression pattern in the human tongue. ${ }^{11} \mathrm{~A}$ short C-terminal peptide fragment of calgranulin $c$ (S100A12) has elicited bactericidal properties against Gram-negative bacteria. ${ }^{22}$ We could demonstrate that keratinocytes release, as the principal E. coli-killing compound, psoriasin in vitro and in vivo. Therefore, psoriasin may be the key to the resistance of human skin toward E. coli, utilizing $\mathrm{Zn}^{2+}$ sequestration as a possible antimicrobial mechanism. ${ }^{12}$ In addition, Yoshio et al. ${ }^{23}$ identified psoriasin in the vernix caseosa, suggesting that it could play a role in the uterus and possibly protect the newborn from $E$. coli infection during birth.

Until now, only little is known about psoriasin expression in the human tongue. One report in 1991 described psoriasin expression in fetal tissues of ear, skin, and tongue as determined by $2 \mathrm{D}$ gel electrophoresis. ${ }^{17}$ Now we provide the first evidence that psoriasin is one of the dominant proteins produced and released by the adult tongue. By immunohistochemistry, we identified psoriasin production in the epithelial layers of the tongue, consistent to the results in the skin. ${ }^{12}$ Psoriasin staining was more intense in the upper, differentiated cell layers than in the suprabasal layers and was identified in the cytoplasm as well as membrane-bound, which might be explained by the redistribution of S100A7 to the cell periphery during cell differentiation. ${ }^{24,25}$ The peripheral distribution observed is important, because psoriasin may be released into saliva, as recently demonstrated by Gläser et al. ${ }^{12}$ for human skin. Intense generalized mucosal expression might indicate a high constitutive expression and/or a permanent stimulation as a defense strategy in a germ-enriched area.

The highest psoriasin expression was found in the anterior part of the tongue with decreasing expression posteriorly. Since the anterior part of the tongue has first contact with microbes and is more vulnerable to surface trauma, it is plausible that this lingual region requires additional protection by high expression of antimicrobial proteins such as psoriasin.

Moreover, psoriasin could not be detected in superficial epithelial cells, which supports the idea that psoriasin is stored and rapidly released to the surface with minimal adherence to the most superficial epithelial cells as suggested by high psoriasin levels that we found in the rinsing fluids of human tongues.

The abundance of psoriasin on the human tongue together with its high antimicrobial activity against $E$. coli strengthens the hypothesis that psoriasin may function as an important factor to control E. coli growth on the tongue surface in vivo. To further analyze the role of psoriasin in the lingual defense system, we repeatedly rinsed a standardized area of the human tongue in healthy volunteers and detected high psoriasin secretion in vivo. Even several times of washing over $24 \mathrm{~h}$ as well as repeated rinsing during a short time period could not significantly reduce the psoriasin concentration, indicating a huge psoriasin production in the upper lingual epithelium, which might be increased in response to permanent microbial and/or inflammatory stress, ${ }^{18}$ as it has been shown for other antimicrobial peptides in the oral cavity. ${ }^{5}$ Interestingly, psoriasin is also secreted and has recently been elicited to absorb $\mathrm{Zn}^{2+}$ ions as a possible antimicrobial defense mechanism against E. coli in the skin. ${ }^{12}$ Although the exact mechanism of psoriasin E. coli-killing activity still remains to be elucidated, $\mathrm{Zn}^{2+}$ deprivation ultimately would affect $\mathrm{Zn}^{2+}$-dependent enzymes, which are essential to protect $E$. coli against oxidative damage by endogenously generated hydrogen peroxide ${ }^{26,27}$ and it is therefore tempting to speculate that psoriasin uses this strategy to be antimicrobial.

In summary, our results indicate an important role of psoria$\sin$ as the principal E. coli-killing antimicrobial protein released by the human tongue. Further investigations are needed to understand the role of psoriasin in the innate lingual defense system.

\section{METHODS}

Isolation and characterization of psoriasin from the human tongue. All human samples were collected in compliance with the laws and guidelines given by the ethic committee of the medical faculty, University of Kiel, Germany, subjected to the 1975 Helsinki Declaration, revised 1983. Lingual tissue was derived from normal individuals posthumously during autopsy after cardiac arrest $(n=17)$. In addition, for all in vivo experiments, healthy volunteers $(n=25)$ were informed in detail and gave written consent.

Only the superficial tissue layers including the epithelia were dissected, and pooled lingual surface tissue ( $85 \mathrm{~g}$ ) was extracted with acidic ethanolic citrate buffer and further purified using a heparin affinity column followed by preparative reversed-phase (C8) HPLC as described before. $^{28}$

Aliquots (10-30 $\mu$ l) of the resulting C8 HPLC fractions were lyophilized, dissolved in $5 \mu \mathrm{l} 0.1 \%(\mathrm{v} / \mathrm{v})$ aqueous acetic acid, and tested for antimicrobial activity against E. coli ATCC 35218 and ATCC 11303 by a radial diffusion assay. ${ }^{29}$ Fractions containing the highest antimicrobial activity against $E$. coli were further purified by $\mathrm{C} 2 / \mathrm{C} 18$ reversed-phase HPLC as previously described. ${ }^{12}$

Fractions containing the highest antimicrobial activity against E. coli were analyzed by electrospray ionization-mass spectrometry, performed in the positive ionization mode with a quadrupol orthogonal accelerating time-of-flight mass spectrometer (QTOF-II-hybrid-mass-spectrometer; Micromass, Manchester, UK). Protein identification was done by peptide mass fingerprinting using tryptic digests. Briefly, lyophilized HPLC fractions containing approximately $2-4 \mu \mathrm{g}$ protein were dissolved in $20 \mu \mathrm{l} \mathrm{NH}{ }_{4} \mathrm{CO}_{3}, \mathrm{pH}$ 8.0. Tryptic digestion was started by adding $50 \mathrm{ng}$ activated (with $1 \%$ acetic acid) trypsin (modified trypsin, sequencing grade; Roche, Mannheim, Germany) and was allowed to proceed for at least $4 \mathrm{~h}$ at $37^{\circ} \mathrm{C}$. Digestion samples were desalted using $10 \mu \mathrm{l} \mathrm{C18} \mathrm{Zip-}$ Tip pipette tips (Millipore, Bedford, MA) and tryptic fragment masses were determined by electrospray ionization-mass spectrometry analyses. Identification of the protein was achieved by analyzing the resulting peptide fragment masses with the peptide mass fingerprinting tool Mascot (www.matrixscience.com).

Immunohistochemistry. To identify the cellular source of psoriasin expression in the tongue, immunohistochemistry with a specific monoclonal anti-psoriasin antibody was performed as previously described. ${ }^{12} \mathrm{~A}$ detection system StreptABComplex/HRP (DAKO, Hamburg, Germany) with DAB (Biogenics, San Ramon, CA) as substrate was used. 


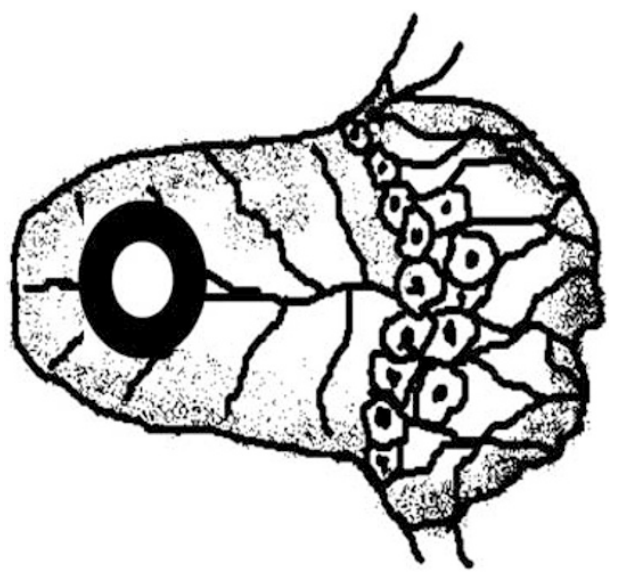

Figure 5 Schematic drawing of the in vivo testing of psoriasin on the tongue. The circle indicates the standardized rinsing area on the surface of the tongue.

Psoriasin ELISA and western blot. For investigation and quantification of psoriasin secretion in human tongue-washing fluids, a psoriasin enzyme-linked immunosorbent assay (ELISA) with two monoclonal antibodies was used as previously described. ${ }^{12}$ Standard curves were generated using natural skin-derived psoriasin and the detection limit of the ELISA was $1 \mathrm{ng} \mathrm{ml}^{-1}$ psoriasin. Specificity testing was performed by western blot analysis as described before ${ }^{12}$ with lingual rinsing fluids derived from different individuals.

In vivo experiments to determine the functional role of psoriasin in the human tongue. To determine the in vivo concentration of psoriasin released on the healthy tongue surface, 25 normal volunteers (average age 35.4; minimum 19, maximum 57; 15 female, 10 male volunteers) were investigated. At the beginning of the experiment, all volunteers irrigated her/his mouth three times with $25 \mathrm{ml}$ normal sterile saline solution to avoid non-lingual psoriasin contamination. A standardized area $\left(50 \mathrm{~mm}^{2}\right)$ of the anterior part of the tongue was prepared by pressing a plastic cylinder on the tongue surface. This area was rinsed in 20 volunteers repeatedly with $900 \mu \mathrm{l} 10 \mathrm{mM}$ sodium phosphate buffer ( $\mathrm{pH} 7.4)$ after $30 \mathrm{~min}, 1,2,6,12$, and $24 \mathrm{~h}(n=20$, Figure 5), whereas the other five volunteers (average age 45.4; minimum 32, maximum 57; two female, three male volunteers) were rinsed 20 times consecutively with $900 \mu \mathrm{l}$ $10 \mathrm{mM}$ sodium phosphate buffer ( $\mathrm{pH} 7.4$ ) on the same area following the three oral irrigations. All individual rinsing fluids were mixed with $100 \mu \mathrm{l}$ $10 \mathrm{mM}$ sodium phosphate buffer ( $\mathrm{pH} 7.4$ ) containing $10 \%$ bovine serum albumin for stabilization and stored at $-80^{\circ} \mathrm{C}$ for further investigations. Quantification of psoriasin concentrations in the tongue rinsing fluids was determined by ELISA.

\section{ACKNOWLEDGMENTS}

We thank H. Janssen, J. Quitzau, M. Brandt (Department of Dermatology, Kiel) for excellent technical assistance. This work was supported by the Deutsche Forschungsgemeinschaft (SFB 617). JH is supported by a Heisenberg fellowship from the Deutsche Forschungsgemeinschaft.

\section{DISCLOSURE}

The authors declared no conflict of interest.

(๐) 2008 Society for Mucosal Immunology

\section{REFERENCES}

1. White, C.B. \& Foshee, W.S. Upper respiratory tract infections in adolescents. Adolesc. Med. 11, 225-249 (2000).

2. Loda, F.A., Glezen, W.P. \& Clyde, W.A. Jr. Respiratory disease in group day care. Pediatrics 49, 428-437 (1972).

3. Quirynen, M. et al. Impact of tongue cleansers on microbial load and taste. J. Clin. Periodontol. 31, 506-510 (2004).
4. Weinberg, A., Krisanaprakornkit, S. \& Dale, B.A. Epithelial antimicrobial peptides: review and significance for oral applications. Crit. Rev. Oral. Biol. Med. 9, 399-414 (1998).

5. Dale, B.A. \& Fredericks, L.P. Antimicrobial peptides in the oral environment: expression and function in health and disease. Curr. Issues Mol. Biol. 7, 119-133 (2005).

6. Harder, J., Meyer-Hoffert, U., Wehkamp, K., Schwichtenberg, L. \& Schröder, J.M. Differential gene induction of human beta-defensins (hBD-1, -2, -3, and -4) in keratinocytes is inhibited by retinoic acid. J. Invest. Dermatol. 123, 522-529 (2004).

7. Schonwetter, B.S., Stolzenberg, E.D. \& Zasloff, M.A. Epithelial antibiotics induced at sites of inflammation. Science 267, 1645-1648 (1995).

8. Schroder, J.M. Epithelial antimicrobial peptides: innate local host response elements. Cell Mol. Life Sci. 56, 32-46 (1999).

9. Diamond, G., Russell, J.P. \& Bevins, C.L. Inducible expression of an antibiotic peptide gene in lipopolysaccharide-challenged tracheal epithelial cells. Proc. Natl. Acad. Sci. USA 93, 5156-5160 (1996).

10. Mathews, M. et al. Production of beta-defensin antimicrobial peptides by the oral mucosa and salivary glands. Infect. Immun. 67, 2740-2745 (1999).

11. Eversole, L.R., Miyasaki, K.T. \& Christensen, R.E. The distribution of the antimicrobial protein, calprotectin, in normal oral keratinocytes. Arch. Oral. Biol. 37, 963-968 (1992).

12. Gläser, R. et al. Antimicrobial psoriasin (S100A7) protects human skin from Escherichia coli infection. Nat. Immunol. 6, 57-64 (2005).

13. Hamilton, W.P., Kim, M. \& Thackston, E.L. Comparison of commercially available Escherichia coli enumeration tests: implications for attaining water quality standards. Water Res. 39, 4869-4878 (2005).

14. Caprioli, A., Morabito, S., Brugere, H. \& Oswald, E. Enterohaemorrhagic Escherichia coli: emerging issues on virulence and modes of transmission. Vet. Res. 36, 289-311 (2005).

15. Tauxe, R.V. Emerging foodborne diseases: an evolving public health challenge. Emerg. Infect. Dis. 3, 425-434 (1997).

16. Todd, E.C. Epidemiology of foodborne diseases: a worldwide review. World Health Stat. Q 50, 30-50 (1997).

17. Madsen, P. et al. Molecular cloning, occurrence, and expression of a novel partially secreted protein 'psoriasin' that is highly up-regulated in psoriatic skin. J. Invest. Dermatol. 97, 701-712 (1991).

18. Eckert, R.L. et al. S100 proteins in the epidermis. J. Invest. Dermatol. 123, 23-33 (2004).

19. Heizmann, C.W., Fritz, G. \& Schafer, B.W. S100 proteins: structure, functions and pathology. Front Biosci. 7, d1356-d1368 (2002).

20. Clohessy, P.A. \& Golden, B.E. Calprotectin-mediated zinc chelation as a biostatic mechanism in host defence. Scand J. Immunol. 42, 551-556 (1995).

21. Sohnle, P.G., Hunter, M.J., Hahn, B. \& Chazin, W.J. Zinc-reversible antimicrobial activity of recombinant calprotectin (migration inhibitory factor-related proteins 8 and 14). J. Infect. Dis. 182, 1272-1275 (2000).

22. Cole, A.M. et al. Calcitermin, a novel antimicrobial peptide isolated from human airway secretions. FEBS Lett. 504, 5-10 (2001).

23. Yoshio, H. et al. Antimicrobial polypeptides of human vernix caseosa and amniotic fluid: implications for newborn innate defense. Pediatr. Res. 53, 211-216 (2003).

24. Broome, A.M., Ryan, D. \& Eckert, R.L. S100 protein subcellular localization during epidermal differentiation and psoriasis. J. Histochem. Cytochem. 51, 675-685 (2003).

25. Ruse, M., Broome, A.M. \& Eckert, R.L. S100A7 (psoriasin) interacts with epidermal fatty acid binding protein and localizes in focal adhesion-like structures in cultured keratinocytes. J. Invest. Dermatol. 121, 132-141 (2003).

26. Imlay, J.A. Pathways of oxidative damage. Annu. Rev. Microbiol. 57, 395-418 (2003).

27. Gort, A.S., Ferber, D.M. \& Imlay, J.A. The regulation and role of the periplasmic copper, zinc superoxide dismutase of Escherichia coli. Mol. Microbiol. 32, 179-191 (1999).

28. Harder, J. \& Schröder, J.M. RNase 7, a novel innate immune defense antimicrobial protein of healthy human skin. J. Biol. Chem. 277, 4677946784 (2002).

29. Steinberg, D.A. \& Lehrer, R.I. Designer assays for antimicrobial peptides. Disputing the 'one-size-fits-all' theory. Methods Mol. Biol. 78, 169-186 (1997). 